\title{
Einführung: Energie - 50 Jahre vorausgedacht
}

\author{
K. Dorfmeister OVE
}

Online publiziert am 26. September 2012

(C) Springer-Verlag 2012

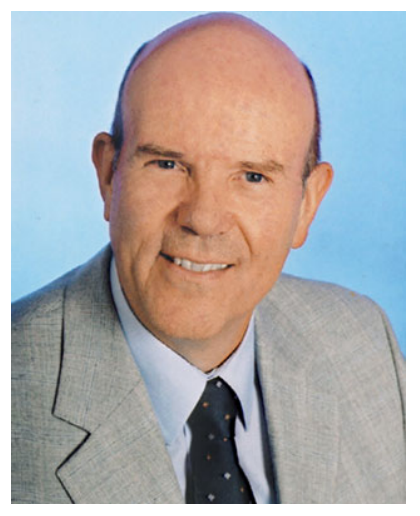

Dipl.-Ing., Dr. Karl Dorfmeister
In der heutigen Zeit ist es sicher nicht leicht, vor allem im Energiebereich ein halbes Jahrhundert vorauszudenken. Visionäre hat es ja immer schon gegeben (in früheren Kulturen waren sie besser als Orakel bekannt), mit mehr oder minder guten Erfolgen, und oft genug mussten Visionen dann später wieder revidiert werden.

Ein kurzer Blick 50 Jahre zurück soll zeigen, wie schwierig Zukunftsvorhersagen sein können:

In Deutschland ist soeben das Kernkraftwerkszeitalter angebrochen, und auch die Schweiz wird sich ein Jahr später für die Kernkraft entscheiden - aus heutiger Sicht dürften die Jahre der Kernkraftwerke in Deutschland und der Schweiz allerdings gezählt sein. Österreich wollte sich der Kernkraftwerkstechnik auch nicht verschließen, hat sich dann vor der Inbetriebnahme allerdings entschieden, endgültig auf die Kernkraftnutzung zu verzichten. Für die Versorgung mit KFZ-Treibstoffen waren keine Probleme absehbar, und wer konnte schon ahnen, dass Erdöl bald als politische Waffe eingesetzt wird? Immerhin schärften die folgenden politischen Aktionen das Bewusstsein, wie abhängig vor allem Europa von der Versorgung mit fossilen Brennstoffen durch andere Länder ist. Der Elektroantrieb für Autos war zwar schon 1900 ein Thema (Radnabenmotor im Lohner Porsche), vor 50 Jahren hat aber niemand mehr daran gedacht. Ein halbes Jahrhundert später vergeht kein Monat, wo nicht ein neues E-Mobil angekündigt wird. Die weitere Zukunft wird zeigen, wann mit praxisgerechten Modellen zu rechnen ist.

Digitale Telefonie? Ein Begriff, den es Anfang der 1960er Jahre einfach noch nicht gab. Die ersten Ansätze für mobile (analoge) Autotelefone waren zwar schon vorhanden, es konnte sich aber niemand die heutige Verbreitung der digitalen Mobiltelefonie vorstellen.

Eng mit den Mobiltelefonen ist heute die Digitalfotografie verbunden (nach dem Motto "kein Handy ohne Kamera"), aber vor 50 Jahren? Erst rund 30 Jahre später ist die erste "richtige" Digitalkamera vorgestellt worden, und seitdem jagt ein neues Kameramodell das andere.

Bleibt für den Kurzrückblick nur noch der PC: Selbst zu Beginn der 1970er Jahre sahen sogar profunde Kenner der damaligen Computerbranche keinen Bedarf an derartigen Geräten für die breite Allgemeinheit. Niemand hätte damals gedacht, dass ohne diese Geräte bzw. deren „Herzen“, die Mikroprozessoren, 50 Jahre später einfach kein Alltagsleben mehr möglich sein wird.

Unter diesen Aspekten scheint es wirklich nicht leicht, belastbare Aussagen über zukünftige technische Entwicklungen, noch dazu auf dem Energiesektor, zu tätigen. Dieser Herausforderung stellen sich aber trotzdem die Energietechnischen Gesellschaften der Electrosuisse, des VDE und des OVE bei ihrer diesjährigen gemeinsamen Fachtagung. Fachkundige Experten aus Forschung, Politik, öffentlicher Verwaltung, Industrie und Energiewirtschaft setzen sich mit den technischen Entwicklungen auf dem Energiesektor der nächsten 50 Jahre auseinander.

Den Experten ist schon heute klar, welche Maßnahmen zu treffen sind, damit nicht eines Tages "das Licht in Europa ausgeht". Der zunehmend aus den Offshore-Windparks im Norden Deutschlands erzeugte Strom kann nur durch entsprechende Netze zu den südlicher gelegenen Verbraucherschwerpunkten und den Pumpspeicherkraftwerken in den Alpen transportiert werden. Können Politik und Marktregeln allein ein Umdenken in der Stromversorgung erzwingen, ohne Berücksichtigung der physikalischen Zusammenhänge?

Die aufkommende E-Mobilität gilt es, unter Berücksichtigung aller Randbedingungen und ohne Euphorie in die vorhandene Versorgungsinfrastruktur einzubinden. Auch für die noch zu entwickelnden elektrischen Verkehrsmittel sind die den Anforderungen entsprechenden Techniken zu verbessern und zu optimieren. Die unterschiedlichen Speichermedien werden dabei eine entscheidende Rolle spielen. Wird die ausschließliche direkte Stromspeicherung in Batterien die beste Lösung bleiben oder wird doch in der Hybridtechnik die Zukunft liegen?

Dies sind wesentliche Kernfragen, aus denen sich viele weitere ergeben und mit denen sich die Gemeinschaftsfachtagung beschäftigt. Ziel ist die Findung seriöser und realistischer Antworten für Gesellschaft, Politik, Industrie und Energiewirtschaft, um einen Leitfaden für die Umsetzung einer bedarfsgerechten und sicheren Stromversorgung für die nächsten 50 Jahre zu finden.

Zum Abschluss der Tagung finden Interessierte die Möglichkeit, im Zuge zweier Exkursionen die Hauptschaltleitung der Austrian Power Grid AG zu besichtigen.

Damit diese Fachtagung einem möglichst großen Teilnehmerkreis zugänglich gemacht werden kann, unterstützt sie die VERBUND AG als Hauptsponsor und weiters die ABB AG, ALSTOM Austria GmbH, ANDRITZ HYDRO GmbH, Austrian Power Grid AG, Energie-Control GmbH, ENERGIE STEIERMARK AG, EW Wels GmbH, Schneider Electric Energy Austria AG, SIEMENS AG Österreich, Sprecher Automation $\mathrm{GmbH}$, TIWAG-Tiroler Wasserkraft AG sowie die Wien Energie Stromnetz $\mathrm{GmbH}$, wofür sich die OGE auf diesem Wege besonders bedankt.

Dorfmeister, Karl, Dipl.-Ing., Dr., Geschäftsführer der Österreichischen Gesellschaft für Energietechnik (OGE) im OVE, Tagungsleiter, Wien, Österreich (E-Mail: Karl.Dorfmeister@verbund.com) 